\title{
Modification of raised cosine weighting functions family
}

\author{
C. Lesnik, A. Kawalec \& J. Pietrasinski \\ Department of Electronics, Military University of Technology, Poland
}

\begin{abstract}
Modification of the known family of raised cosine weighting functions with the power of $n$ by using its convolution operation with an auxiliary rectangular window having variable duration time is considered in the paper. Such a modified functions family was derived in a general and exact form for the defined weighting function in a time and frequency domain as well. It was done with the help of the convolution windows constant - length type preparation technique. The derived weighting function was applied for radar chirp signals synthesis with nonlinear frequency modulation (NLFM). Chosen examples of the simulation research of the discussed modified weighting functions family features and radar signals of NLFM type synthesised thanks to it are presented in the paper.
\end{abstract}

Keywords: weighting functions, radar signal synthesis, nonlinear frequency modulation signal, mainlobe, sidelobe, matched filtration.

\section{Introduction}

Radar signals synthesis is one of the most important problems of modern radiolocation. These signals transmitted to observed space should have very specific features.

The so-called matched filter is a very specific part of a radar receiver. Its main task is to maximize SNR (Signal to Noise Ratio) at its output. In this way radar range may be maximized too. In the case of radar chirp signals transmission an echo signal observed at the receiver output has a shape of the very short pulse. Thanks to it radar range resolution may be good. A product of a received signal matched filtration is an output signal having mainlobe and sidelobes. Presence of sidelobes is of course an unwanted effect because it causes weak echo signals detection difficulty. 
An effective technique of sidelobes attenuation is suitable weighting function application. These are so called weighting windows that modify the matched filter transmittance. A resulted filter mismatch is an additional effect of the weighting procedure. It leads to the worse value of the output SNR and in this way it causes radar range shortening.

Application of radar signals of NLFM type is a lossless technique of sidelobes attenuation. The principle of stationary phase is used for the NLFM signals synthesis, detailed elsewhere in [1-3]. In such an approach suitable weighting functions are applied too. As a result of principle of stationary phase using unwanted accumulation of numerical errors can be observed. In order to its decrease the knowledge concerning exact, analytical form of the weighting function applied for the signal synthesis is desired.

There are several known techniques used for weighting functions synthesis having expected features important from the point of view of application. One of them is based on convolution operation. To be more precise, there are known methods, such as: time convolution of parent windows, described by Harris [4] and Nuttall [5], multiple time convolution of rectangular windows, described by Wen [6] and Dai and Gretsch [7] or multiple time auto and cross convolution of other well known windows, described by Reljin et al. [8,9]. These so called convolution windows have greater sidelobe attenuation and their fast decay. Unfortunately the output signal mainlobe width increases.

Modification of the known family of the raised cosine weighting functions by using convolution operation of this functions with an auxiliary rectangular window having variable duration time is considered in the paper. It enables finetuning of the weighting function properties. The exact analytical formula of the modified function was derived in time and frequency domain.

The paper is organized as follows. The known idea of convolution weighting function preparation is presented in section 2 as an introductory remark. The exact closed formula of the modified raised cosine weighting functions family in the continuous domain of time and frequency is derived in section 3. Examples of chosen features of the modified raised cosine weighting functions family are discussed in section 4. There is an example of its application for radar signal NLFM type synthesis too. A few sentences of conclusion are written in section 5.

\section{Convolution weighting function design}

General form of weighting function with finite time duration $w_{T}(t)$ can be described as a product as follows

where:

$$
w_{T}(t)=w(t) r_{T}(t)
$$

$w(t)$ - considered unlimited time function,

$r_{T}(t)$ - unitary rectangular window as follows: 


$$
r_{T}(t)=\operatorname{rect}\left(\frac{t}{T}\right)=\left\{\begin{array}{ll}
1 & \text { for }|t| \leq \frac{T}{2} \\
0 & \text { for elsewhere } t
\end{array}, T>0,\right.
$$

$T$ - rectangular window time duration.

Equation (1) is the equivalent of the convolution operation in the frequency domain

$$
W_{T}(\omega)=\frac{1}{2 \pi}\left[W(\omega) * R_{T}(\omega)\right]
$$

where: $W(\omega)$ and $R_{T}(\omega)$ are Fourier transforms of the $w(t)$ and $r_{T}(t)$ respectively.

Let us consider weighting function modification $w_{T}(t)$ described by eqn (3) that is based on its convolution with auxiliary rectangular window $r_{\tau}(t)$ having variable duration time $\tau$

$$
w_{\tau}(t)=w_{T}(t) * r_{\tau}(t)=\left[w(t) r_{T}(t)\right] * r_{\tau}(t),
$$

where $w_{\tau}(t)$ - modified weighting function.

The auxiliary rectangular window $r_{\tau}(t)$ is defined as follows

$$
r_{\tau}(t)=\operatorname{rect}\left(\frac{t}{\tau}\right)=\left\{\begin{array}{ll}
\frac{1}{\tau} & \text { for }|t| \leq \frac{\tau}{2} \\
0 & \text { for elsewhere } \mathrm{t}
\end{array}, \quad \tau>0,\right.
$$

where $\tau$ - variable duration time of the auxiliary rectangular window. A factor $1 / \tau$ causes that the weighting function max value described by eqn (4) is independent of the auxiliary rectangular window duration time. It results from the fact that the area under $r_{\tau}(t)$ is unitary, described by Brandwood [10]. The convolution operation described by eqn (4) equals formula as follows in the frequency domain

$$
W_{\tau}(\omega)=\frac{1}{2 \pi}\left[W(\omega) * R_{T}(\omega)\right] R_{\tau}(\omega),
$$

where: $W_{\tau}(\omega)$ and $R_{\tau}(\omega)$ are the Fourier transforms of the $w_{\tau}(t)$ and $r_{\tau}(t)$ respectively.

As a result of convolution operation modified weighting function $w_{\tau}(t)$ is different from zero within an interval from $-(T+\tau) / 2$ up to $(T+\tau) / 2$ and its duration time is $(T+\tau)$. In order to keep constant the final weighting function duration time (it equals $T$ ) there is necessity for suitable scaling of eqns (4) and (6) in time and frequency according to the Fourier transformation scaling property 


$$
x\left(\frac{t}{a}\right) \leftrightarrow|a| X(a \omega),
$$

where factor of scale change is defined as follows

$$
a=\frac{T}{T+\tau} .
$$

As a result of eqns (4) and (6) scaling the general form of the modified weighting functions in the time and frequency domain are as follows:

$$
\begin{aligned}
w_{\tau}(t) & =\left[w\left(\frac{t}{a}\right) r_{T}\left(\frac{t}{a}\right)\right] * r_{\tau}\left(\frac{t}{a}\right), \\
W_{\tau}(\omega) & =\frac{a}{2 \pi}\left[W(a \omega) * R_{T}(a \omega)\right] R_{\tau}(a \omega) .
\end{aligned}
$$

Scaled modified weighting function described by eqn (7) is different from zero within the interval from $-T / 2$ up to $-T / 2$ and their duration time is $T$.

The window function received in this way is called constant-length CON window and it is described by Reljin et al. [9].

The presented method will be applied for known and very useful (among others for synthesis of the radar and sonar signals with nonlinear frequency modulation, NLFM) family of cosine windows.

\section{Modification of raised cosine weighting functions family - general solution}

Let us consider cosine window family with power of $n$ (raised cosine with power of $n$ ) having a general form as follows

$$
w_{T}^{(n)}(t)=\left[k+(1-k) \cos ^{n}\left(\pi \frac{t}{T}\right)\right] \operatorname{rect}\left(\frac{t}{T}\right),
$$

where:

$k \quad$ - real parameter of the function, $k \in\langle 0,1\rangle$,

$n \quad$ - integer parameter of the function, $n \geq 1$,

$T$ - weighting function duration time.

As a result of known trigonometric identities application the general description of the function described by eqn (9) with the $n$-th power was achieved

$$
w_{T}^{(n)}(t)=\left[A+B \sum_{i=0}^{s-1}\left(\begin{array}{l}
n \\
i
\end{array}\right) \cos n_{i} \pi \frac{t}{T}\right] \operatorname{rect}\left(\frac{t}{T}\right),
$$


where:

$$
\begin{aligned}
& s=\left\lfloor\frac{n+1}{2}\right\rfloor,\lfloor x\rfloor \text { the biggest integer not higher than } x, \\
& A= \begin{cases}k & \text { for odd } n, \quad n=1,3,5, \ldots \\
k+\frac{1-k}{2^{n}}\left(\begin{array}{c}
n \\
n / 2
\end{array}\right) & \text { for even } n, \quad n=2,4,6, \ldots\end{cases} \\
& B=\frac{1-k}{2^{n-1}}, \\
& n_{i}=\left(\begin{array}{l}
n-2 i), \\
\left(\begin{array}{l}
x \\
y
\end{array}\right), x \geq y-\text { binomial coefficients. }
\end{array}\right.
\end{aligned}
$$

As a result in the general case the weighting function described by eqn (10) in the time domain is a sum of a constant component and cosine functions limited in time and scaled in amplitude. Their period is a total submultiple of the fundamental period that equals $2 T$.

Considering the weighting function general description presented in the eqn (10) in the frequency domain and based on the eqn (6) one can obtain

$$
W_{T}^{(n)}(\omega)=\frac{1}{2 \pi}\left[W^{(n)}(\omega) * R_{T}(\omega)\right] .
$$

Using Fourier transforms pairs

$$
1 \leftrightarrow 2 \pi \delta(\omega), \quad \cos \omega_{0} t \leftrightarrow \pi \delta\left(\omega-\omega_{0}\right)+\pi \delta\left(\omega+\omega_{0}\right)
$$

one can achieve

$$
W^{(n)}(\omega)=A 2 \pi \delta(\omega)+B 2 \pi \sum_{i=0}^{s-1}\left(\begin{array}{l}
n \\
i
\end{array}\right)\left[\delta\left(\omega-n_{i} \frac{\pi}{T}\right)+\delta\left(\omega+n_{i} \frac{\pi}{T}\right)\right] .
$$

After Fourier transform of the rectangular window application

$$
\operatorname{rect}\left(\frac{t}{T}\right) \leftrightarrow T \mathrm{Sa} \frac{\omega T}{2}
$$

where $\mathrm{Sa}($.$) denotes a function in a shape of \mathrm{Sa}(x)=(\sin x) / x$, and using delta distribution properties it is possible to achieve the final, general form of the cosine functions family with power of $n$ in the frequency domain

$$
W_{T}^{(n)}(\omega)=A T \operatorname{Sa} \frac{\omega T}{2}+\frac{B}{2} T \sum_{i=0}^{s-1}\left(\begin{array}{c}
n \\
i
\end{array}\right)\left\{\mathrm{Sa}\left[\left(\omega-n_{i} \frac{\pi}{T}\right) \frac{T}{2}\right]+\mathrm{Sa}\left[\left(\omega+n_{i} \frac{\pi}{T}\right) \frac{T}{2}\right]\right\} .
$$

This function is a sum of functions of the $(\sin x) / x$ type that parameters and location on the radian frequency axis depend on the power $n$ and the window duration time $T$. 
The general form of the modified weighting function in the time domain, defined for separate time intervals was solved thanks to convolution operation of the function presented in the eqn (10) with the auxiliary rectangular window described by eqn (5):

$$
\begin{gathered}
\text { for }-\frac{T}{2}-\frac{\tau}{2} \leq t<-\frac{T}{2}+\frac{\tau}{2} \\
\qquad \begin{array}{c}
w_{\tau}^{(n)}(t)=\left\{\frac{A}{2}\left(1+\frac{T}{\tau}\right)+B \frac{T}{\tau \pi} \sum_{i=0}^{s-1} \frac{\left(\begin{array}{c}
n \\
i
\end{array}\right)}{n_{i}} \sin n_{i} \frac{\pi}{2}+\right. \\
\left.+\frac{A}{\tau} t+B \frac{T}{\tau \pi} \sum_{i=0}^{s-1} \frac{\left(\begin{array}{l}
n \\
i
\end{array}\right)}{n_{i}} \sin \left[n_{i} \frac{\pi}{T}\left(t+\frac{\tau}{2}\right)\right]\right\} \frac{1}{N_{\tau}} \\
\text { for }-\frac{T-\tau}{2} \leq t<\frac{T-\tau}{2} \\
w_{\tau}^{(n)}(t)=\frac{A}{N_{\tau}}+\frac{B}{N_{\tau}} \sum_{i=0}^{s-1}\left(\begin{array}{l}
n \\
i
\end{array}\right) \operatorname{Sa}\left(n_{i} \frac{\pi}{T} \frac{\tau}{2}\right) \cos \left(n_{i} \frac{\pi}{T} t\right),
\end{array}
\end{gathered}
$$

for $\frac{T-\tau}{2} \leq t \leq \frac{T+\tau}{2}$

$$
\begin{aligned}
& w_{\tau}^{(n)}(t)=\left\{\frac{A}{2}\left(1+\frac{T}{\tau}\right)+B \frac{T}{\tau \pi} \sum_{i=0}^{s-1} \frac{\left(\begin{array}{l}
n \\
i
\end{array}\right)}{n_{i}} \sin n_{i} \frac{\pi}{2}-\right. \\
&\left.-\frac{A}{\tau} t-B \frac{T}{\tau \pi} \sum_{i=0}^{s-1} \frac{\left(\begin{array}{l}
n \\
i
\end{array}\right)}{n_{i}} \sin \left[n_{i} \frac{\pi}{T}\left(t-\frac{\tau}{2}\right)\right]\right\} \frac{1}{N_{\tau}}
\end{aligned}
$$

In order to fulfil the condition $w_{\tau}^{(n)}(0)=1$ in eqns (14), (15) and (16) normalization operation was done into the coefficient $N_{\tau}$ that depends on $\tau$

$$
N_{\tau}=A+B \sum_{i=0}^{s-1}\left(\begin{array}{l}
n \\
i
\end{array}\right) \operatorname{Sa}\left(n_{i} \frac{\pi}{T} \frac{\tau}{2}\right)
$$

After scaling in time by using coefficient

$$
a=\frac{T}{T+\tau},
$$


the time interval borders and the final general form of the modified weighting function in the time domain are as follows:

$$
\begin{aligned}
& \text { for }-\frac{T}{2} \leq t<-\frac{(T-\tau) T}{2(T+\tau)} \\
& w_{\tau}^{(n)}(t)=\left\{\frac{A}{2}\left(1+\frac{T}{\tau}\right)+B \frac{T}{\tau \pi} \sum_{i=0}^{s-1} \frac{\left(\begin{array}{l}
n \\
i
\end{array}\right)}{n_{i}} \sin n_{i} \frac{\pi}{2}+\right. \\
& \left.+\frac{A}{\tau} \frac{t}{a}+B \frac{T}{\tau \pi} \sum_{i=0}^{s-1} \frac{\left(\begin{array}{l}
n \\
i
\end{array}\right)}{n_{i}} \sin \left[n_{i} \frac{\pi}{T}\left(\frac{t}{a}+\frac{\tau}{2}\right)\right]\right\} \frac{1}{N_{\tau}} \\
& w_{\tau}^{(n)}(t)=\frac{A}{N_{\tau}}+\frac{B}{N_{\tau}} \sum_{i=0}^{s-1}\left(\begin{array}{l}
n \\
i
\end{array}\right) \operatorname{Sa}\left(n_{i} \frac{\pi}{T} \frac{\tau}{2}\right) \cos \left(n_{i} \frac{\pi}{T} \frac{t}{a}\right),
\end{aligned}
$$

for $\frac{(T-\tau) T}{2(T+\tau)} \leq t<\frac{T}{2}$

$$
\begin{aligned}
& w_{\tau}^{(n)}(t)=\left\{\frac{A}{2}\left(1+\frac{T}{\tau}\right)+B \frac{T}{\tau \pi} \sum_{i=0}^{s-1} \frac{\left(\begin{array}{c}
n \\
i
\end{array}\right)}{n_{i}} \sin n_{i} \frac{\pi}{2}-\right. \\
& \left.-\frac{A}{\tau} \frac{t}{a}-B \frac{T}{\tau \pi} \sum_{i=0}^{s-1} \frac{\left(\begin{array}{l}
n \\
i
\end{array}\right)}{n_{i}} \sin \left[n_{i} \frac{\pi}{T}\left(\frac{t}{a}-\frac{\tau}{2}\right)\right]\right\} \frac{1}{N_{\tau}}
\end{aligned} .
$$

The normalization coefficient described by eqn (17) is the same.

From the eqns (18), (19) and (20) results that the modified weighting function slopes in the time domain are created by the sum of the constant component, linear function and sine functions. Modification of the weighting function in its central part means its scaling in amplitude.

As a result of the modified weighting function exact version analyse in the frequency domain and based on the eqn (6) one can obtain

$$
W_{\tau}^{(n)}(\omega)=W_{T}^{(n)}(\omega) R_{\tau}(\omega)
$$


Using Fourier transformation of the auxiliary rectangular window $R_{\tau}(\omega)$ and eqn (13) and after the normalizing coefficient application $N_{\tau}$ one can achieve

$$
\begin{aligned}
W_{T}^{(n)}(\omega)= & \frac{T}{N_{\tau}} \mathrm{Sa} \frac{\omega \tau}{2} A \mathrm{Sa} \frac{\omega T}{2}+\frac{T}{N_{\tau}} \mathrm{Sa} \frac{\omega \tau}{2} \frac{B}{2} \sum_{i=0}^{s-1}\left(\begin{array}{c}
n \\
i
\end{array}\right) \mathrm{Sa}\left[\left(\omega-n_{i} \frac{\pi}{T}\right) \frac{T}{2}\right]+ \\
& +\frac{T}{N_{\tau}} \mathrm{Sa} \frac{\omega \tau}{2} \frac{B}{2} \sum_{i=0}^{s-1}\left(\begin{array}{c}
n \\
i
\end{array}\right) \mathrm{Sa}\left[\left(\omega+n_{i} \frac{\pi}{T}\right) \frac{T}{2}\right]
\end{aligned}
$$

The final general description of the modified weighting function after scaling operation using is as follows

$$
\begin{aligned}
W_{T}^{(n)}(\omega)= & \frac{T a}{N_{\tau}} \mathrm{Sa} \frac{a \omega \tau}{2} A \mathrm{Sa} \frac{a \omega T}{2}+\frac{T a}{N_{\tau}} \mathrm{Sa} \frac{a \omega \tau}{2} \frac{B}{2} \sum_{i=0}^{s-1}\left(\begin{array}{c}
n \\
i
\end{array}\right) \mathrm{Sa}\left[\left(\omega-n_{i} \frac{\pi}{T a}\right) \frac{T a}{2}\right]+ \\
& +\frac{T}{N_{\tau}} \mathrm{Sa} \frac{a \omega \tau}{2} \frac{B}{2} \sum_{i=0}^{s-1}\left(\begin{array}{c}
n \\
i
\end{array}\right) \mathrm{Sa}\left[\left(\omega+n_{i} \frac{\pi}{T a}\right) \frac{T a}{2}\right]
\end{aligned}
$$

From the eqn (23) results that the auxiliary weighting window is responsible for amplitude modulation of the modified weighting function separate components and it cause changes of their lobes width and location on the radial frequency axis.

\section{Simulation results}

A simulation model of the modified weighting functions generator and the pulse radar signal generator was prepared for testing. It was done in LabWindows CVI (National Instruments) environment. Thanks to the model both weighting functions and generated radar NLFM signals features tests are possible. The influence of the duration time of the auxiliary rectangular window on the generated signals characteristics was the main task of research. As a result a number of characteristics were obtained. A few of them are presented below.

Normalized magnitude spectra of the modified weighting function for different value of the power of $n$ is presented on fig. 1. Values of the parameters $k$ and $\tau$ were fund in the iterative way in order to achieve min level of sidelobes. Normalized magnitude spectra of the non-modified weighting function and rectangular window are presented too on common figures for comparison. The first one was prepared for the same value of the parameter $n$ and for optimum value of the parameter $k$ that gives the minimum level of sidelobes.

A conclusion can be drawn that thanks to the modification operation the sidelobes level in dependence on the $n$ value, decreases from about $14 \mathrm{~dB}$ up to $27 \mathrm{~dB}$ with relation to the case of the non-modified weighting function. The sidelobes level attenuation was achieved at the cost of the mainlobe broadening.

For example, comparable sidelobes level can be obtained for the modified window with $n=2$ and the non-modified window with $n=6$, but in the second case the mainlobe width at $-3 \mathrm{~dB}$ level is 1.35 times wider. 

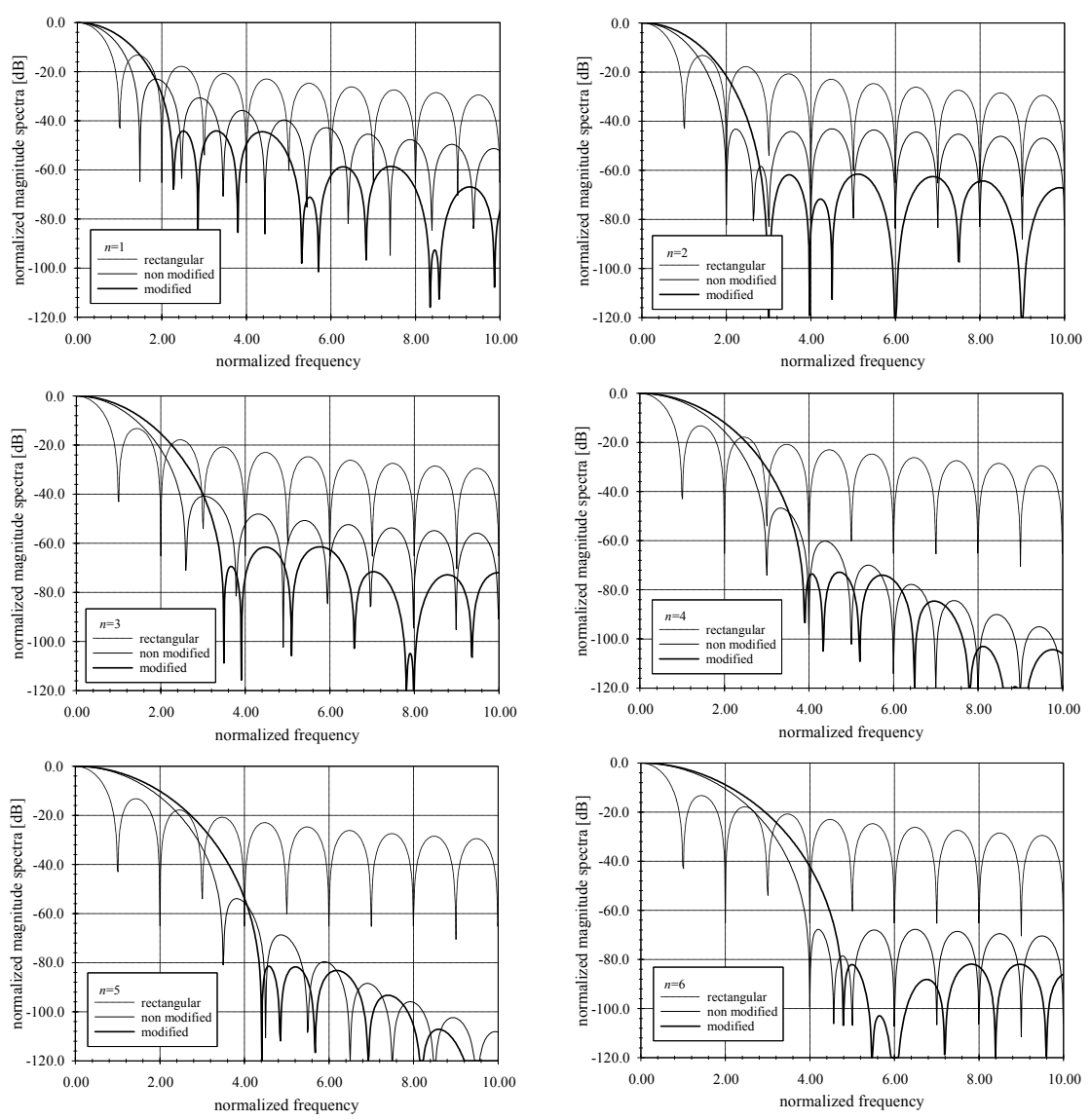

Figure 1: Normalized magnitude spectrum of the modified, non-modified, and rectangular windows for different $n$.

Design of the transmitted NLFM radar signal was done in the next research step. The matched filter output signal is given by the input filter signal autocorrelation function. The signal autocorrelation function is determined by the inverse Fourier transform of the energy spectral density. So to generate a NLFM signal with uniform amplitude one can use the signal with the magnitude spectrum described by a square root of the weighting function, raised cosine with power of $n$ for example. Finding the nonlinear frequency law using the stationary-phase principle was suggested by Fowle [1], Cook and Bernfeld [2].

This method was applied in this paper. As a result some characteristics were obtained. A number of them are presented here. Normalized magnitude of the NLFM signal observed at the matched filter output versus normalized to pulse duration time are depicted in fig. 2 and fig. 3. 
The characteristics are shown for the magnitude spectrum described by nonmodified and modified weighting function and for two different values of the $B T$ product.
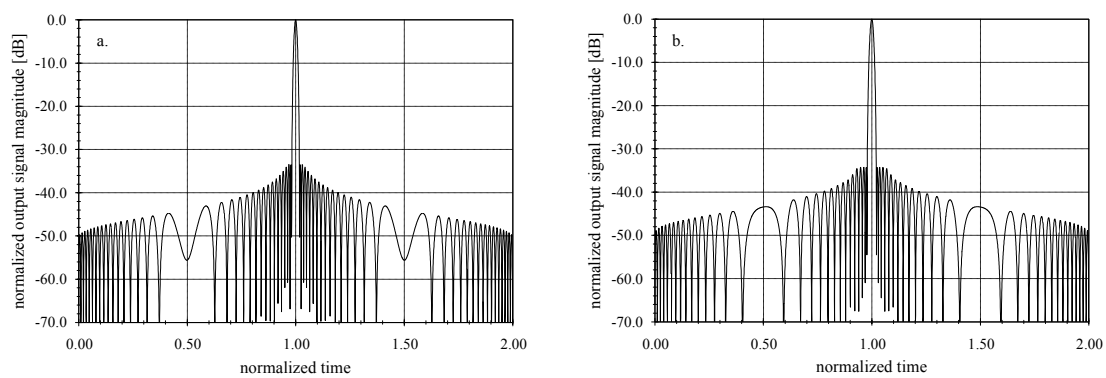

Figure 2: $\quad$ Normalized magnitude output matched filter signal for the nonmodified (a. $k=0.113, \tau=0$ ) and modified (b. $k=0.2153$, $\tau=0.535 T$ ) weighting function, and $B T=100$.
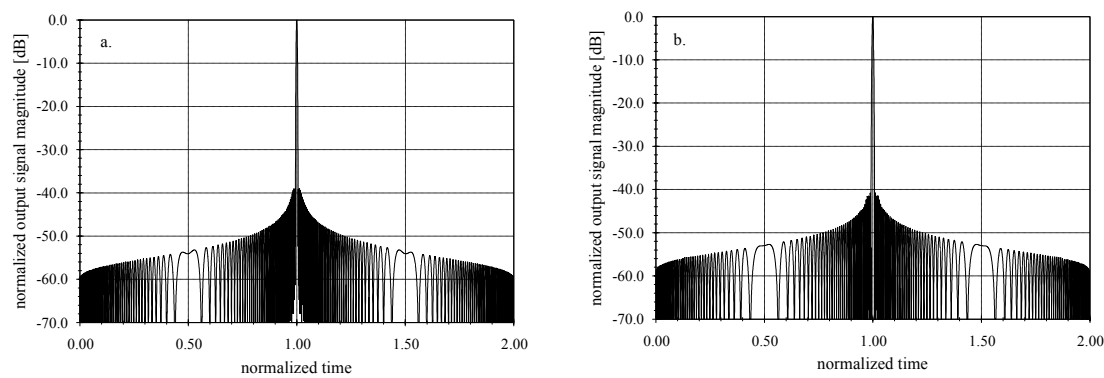

Figure 3: Normalized magnitude output matched filter signal for the non modified (a. $k=0.09, \tau=0$ ) and modified (b. $k=0.094$, $\tau=0.415 T$ ) weighting function, $B T=100$.

As a result of the recursive finding of the minimum sidelobes level by values of the $k$ and $\tau$ parameters changing the $0.8 \mathrm{~dB}$ improvement of this level were obtained for $B T=100$ and $1.8 \mathrm{~dB}$ for $B T=300$ respectively.

\section{Conclusion}

The main goal of the paper is derivation of the general, analytical formula of the modified weighting function and its application for NLFM type radar signals synthesis. The modification essence is the known weighting functions family of raised cosine type convolution with the auxiliary rectangular window having variable changeably duration time. The rectangular auxiliary window duration time selection gives the opportunity for its features fine tuning.

As a result decrease of sidelobes level from $14 \mathrm{~dB}$ up to $27 \mathrm{~dB}$, with simultaneous and expected mainlobe width increase for chosen parameters was 
achieved. Moreover the NLFM radar signal parameters improvement was noticed at the output of the matched filter. The scale of this effect is relatively low and it depends on the $B T$ product.

It is worth to add that the described method may be applied for fine tuning other type of weighting functions.

The modified weighting function described in the paper may be used not only for radar signal synthesis but for harmonic analysis and filter design too.

\section{Acknowledgements}

This work was supported by the National Center for Research and Development for the years 2007-2010 under Commissioned Research Project PBZ-MNiSWDBO-04/I/2007.

\section{References}

[1] Fowle, E.N., The Design of FM Pulse Compression Signals. IEEE Transaction on Information Theory, IT-10, pp. 61-67, 1964.

[2] Cook, C.E. \& Bernfeld, M., Radar Signals, An Introduction to Theory and Application, Artech House, Boston and London, pp. 34-56, 1993.

[3] Levanon, N. \& Mozeson, E., Radar Signals, John Wiley \& Sons, Hoboken, pp. 87-88, 92, 129, 2004.

[4] Harris, F.J., On the Use of Windows for Harmonic Analysis with the Discrete Fourier Transform, Proceedings of the IEEE, 66, pp. 51-83, 1978.

[5] Nuttall, A.H., Some Windows with Very Good Sidelobe Behaviour, IEEE Transaction on Acoustics, Speech, and Signal Processing, ASSP-29, pp. 84-91, 1981.

[6] Wen, P., A Fast and High-Precision Measurement of Distorted Power Based on Digital Filtering Techniques, IEEE Transaction on Instrumentation and Measurement, 41, pp. 403-406, 1992.

[7] Dai, X. \& Gretsch, R., Quasi-Synchronous Sampling Algorithm and its Applications, IEEE Transaction on Instrumentation and Measurement, 43, pp. 204-209, 1994.

[8] Reljin, I.S, Reljin, B.D., Papić, V.D. \& Kostić, P., New Window Functions Generated by Means of Time Convolution - Spectral Leakage Error, Proc. of the 9th Conference MELECON, Tel Aviv, pp. 878-881, 1998.

[9] Reljin, I.S, Reljin, B.D. \& Papić, V.D., Extremely Flat-Top Windows for Harmonic Analysis, IEEE Transaction on Instrumentation and Measurement, 56, pp. 1025-1041, 2007.

[10] Brandwood D., Fourier Transforms in Radar and Signal Processing. Artech House, Boston and London, pp. 40-53, 102-105, 2003. 